\title{
Se projeter vers demain
}

\section{Michel Matter}

Dr méd., membre du Comité central de la FMH, responsable du département Prestations et développement professionnel

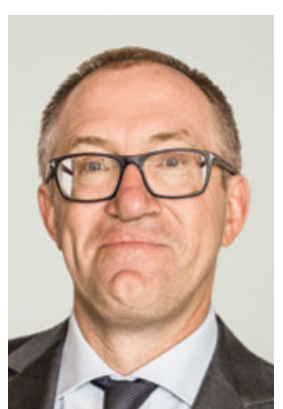

La médecine comme nous la pratiquons actuellement va évoluer et c'est à la fois un bien et une évidence. C'est le rythme de la transformation numérique qui risque d'être un réveil brutal et une révolution. Il n'y a pas un mois sans une conférence sur la digitalisation, l'intelligence artificielle, l'ubérisation de la médecine, le suivi connecté des données médicales des citoyens, les walk-in cliniques avec leurs soins immédiats et la santé personnalisée basée sur une médecine prédictive, participative et préventive. Cette transformation devra s'établir dans le respect des conditions de travail car il est essentiel de veiller à ce que tous les professionnels de la santé n'entrent pas dans un épuisement manifeste en raison de l'absence de réelles stratégies et organisations centrées sur l'activité quotidienne auprès et en l'absence du patient.

La médecine doit prendre un véritable virage. La numérisation est en route et la collection des données, le Big Data, déploie ses ailes et crée les empires de demain. C'est là que la FMH doit rester le cœur de notre organisation professionnelle tant sur la réflexion, les actions à mener que sur la défense de valeurs communes. Les intérêts particuliers seront nombreux, les opportunités de développement seront innombrables, mais tout devra se faire dans un cadre défini dans le respect de l'intérêt de tous.

\section{La médecine doit prendre un véritable virage.}

Nous connaîtrons une médecine sans douleurs grâce aux progrès palpables et promis par l'industrie pharmaceutique. La génomique et par extension la science basée sur l'étude de nos lieux de vie, de nos habitudes alimentaires et comportementales permettront une médecine personnalisée et prédictive. Arriver à individualiser les approches cliniques et thérapeutiques est l'un des buts recherchés, mais cela devra obligatoirement se faire dans une médecine ouverte aux échanges avec l'ensemble des professions qui entourent et soignent le patient. La coordination des soins et l'interprofessionnalité sont et seront l'évidence. La qualité de la prise en charge médicale devra être soutenue avec conviction. L'humain devra rester au centre de nos préoccupations.

L'innovation devra également être pleinement encouragée car la chirurgie est un processus continu. Le soutien au progrès médico-chirurgical doit passer par une

\section{Nous ferons entendre notre voix, celle des} médecins, et nous bougerons nos lignes pour le bien de nos conditions de travail.

volonté politique clairement affichée. La formation des plus jeunes devra être soutenue financièrement et les échanges entre les milieux universitaires et les centres médicaux renforcés. Le financement des soins devra passer par l'uniformisation des prestations de santé ambulatoires et stationnaires.

2018 sera une année charnière: un nouveau tarif sera imposé jusqu'à l'acceptation du TARCO par l'ensemble des médecins puis des partenaires, une volonté parlementaire de mettre fin à l'obligation de contracter contre laquelle nous devrons faire appel au souverain par référendum ou encore le budget global qui promet le rationnement, la médecine à deux vitesses et la mainmise étatique sur la santé. Nous ferons entendre notre voix, celle des médecins, et nous bougerons nos lignes pour le bien de nos conditions de travail mais également pour assurer une médecine de qualité à nos patients.

Etre à la tête du département Prestations et développement professionnel est un véritable challenge tant ce département reste à définir et est en étroite collaboration avec les autres départements. Il permet de se projeter vers cette médecine en révolution où le rythme du changement de paradigme s'accélère chaque jour mais où l'essentiel doit rester l'humain. Je remercie le Dr Remo Osterwalder pour son travail important à la tête de ce département car il aura su montrer la voie et se tourner vers demain. 